\title{
Multilinguales
}

\section{L'écriture fragmentaire : une destruction d'une perspective unitaire du récit dans Terrasse à Rome de Pascal Quignard}

Fragmental writing: a destruction of a narrative unitary perspective in Terrasse à Rome by Pascal Quignard

$$
\text { كينيار الكتابة المجزأة: تدمير منظور وحدوي للرواية في شرفة في روما لباسكال }
$$

\section{Nabila Bekhedidja}

\section{OpenEdition}

\section{Journals}

Édition électronique

URL : http://journals.openedition.org/multilinguales/1063

DOI : 10.4000/multilinguales. 1063

ISSN : 2335-1853

Éditeur

Université Abderrahmane Mira - Bejaia

Référence électronique

Nabila Bekhedidja, «L'écriture fragmentaire : une destruction d'une perspective unitaire du récit dans Terrasse à Rome de Pascal Quignard », Multilinguales [En ligne], 9 | 2018, mis en ligne le 01 juin 2018, consulté le 17 septembre 2019. URL : http://journals.openedition.org/multilinguales/1063 ; DOI : 10.4000/multilinguales. 1063

Ce document a été généré automatiquement le 17 septembre 2019



Multilinguales est mise à disposition selon les termes de la Licence Creative Commons Attribution Pas d'Utilisation Commerciale - Pas de Modification 4.0 International 


\section{L'écriture fragmentaire : une destruction d'une perspective unitaire du récit dans Terrasse à Rome de Pascal Quignard}

Fragmental writing: a destruction of a narrative unitary perspective in Terrasse à Rome by Pascal Quignard كينيار الكتابة المجزأة:تدمير منظور وحدوي للرواية في شرفة في روما لباسكال Nabila Bekhedidja

1 Terrasse à Rome est publié en 2000, raconte la vie d'un jeune homme graveur qui s'appelle Geoffroy Meaume. En 1639, à Bruges, Meaume, un eau -Fortier tombe amoureux de Nanni Veet Jakobsz, une jeune fille de 18 ans. Les deux tourtereaux se feront, néanmoins surprendre sur le fait par Valancre, le fiancé de l'adolescente. Fou de jalousie Valancre jette à la figure de Meaume une fiole d'eau forte. Nanni reçoit quelques éclaboussures sur la main mais Meaume est à jamais défiguré par l'acide.

2 Meaume nous livre au fil des chapitres souvent très courts, sans lien nécessaire les uns avec les autres, ses réflexions d'artiste, d'homme blessé, d'homme amoureux, d'ami trahi, de créateur au sommet de son art et de disciple des plus grands de son temps.

3 Terrasse à Rome est écrit en gros caractères, très aérés car il y a plus de vide et d'espace que de plein dans ce texte. Les chapitres sont brefs, courts, les uns destinés au récit, les autres à des pensées théoriques et puis ceux qui sont uniquement des descriptions de gravures, tout cela forme un tout ou l'eau forte sert de métaphore à l'écriture, à ce difficile art d'écrire et à employer les mots. Ainsi, la composition de ce roman peut laisser le lecteur perplexe: l'ordre chronologique n'est pas respecté, l'histoire est fragmentée et disloquée. Cette composition donne l'impression d'une errance, d'un puzzle où seuls comptent des morceaux isolés, des tranches de vie. Ce qui déroute le lecteur dans cette absence d'ordre chronologique laisse mieux percevoir le chaos d'une 
existence. Ce choix narratif rend compte de l'activité créatrice d'un individu (Meaume) et de son art (peintre).

4 Pascal Quignard exprime dans son roman le désir de réfléchir sur l'acte créateur et l'écriture romanesque en modifiant le système traditionnel de la narration. D'ailleurs, la subversion des formes de ce récit a rebuté de nombreux lecteurs, d'autant plus qu'elle porte en général sur plusieurs aspects de la narration. En ce qui concerne l'intrigue, elle est métamorphosée par le bouleversement imposé à la chronologie et la prolifération des récits. Les ruptures dans la logique de l'enchaînement et les effets de déréalisation systématique ne peuvent que déconcerter un public habitué à adhérer à ce qu'il lit.

5 En effet, Pascal Quignard nous présente un roman disloqué, fragmenté, voire hybride. Le récit lui-même, en plus de se voir fragmenté dans sa disposition et son organisation, est en proie à une fragmentation narrative. Ainsi, les divisions habituelles en chapitres laissent plutôt place à des petits paragraphes séparés les uns des autres. Il arrive parfois qu'un paragraphe solitaire prenne toute la place, isolé sur une page. Aucune règle quantitative ne parait régir la division, les longueurs variant selon les pages. Le fragment se donne parfois comme un récit en soi (ASSELIN, $2003: 153$ ).

6 Le fragment dans Terrasse à Rome, confère au texte, un effet de découpage ou de rapiéçage.

Dès lors notre questionnement est le suivant :

Comment lire ce roman hybride?

Quelles sont les modalités narratives employées par Pascal Quignard pour raconter cette histoire?

Pourquoi les mêmes fragments se répètent-ils dans le roman? Peut-on parler d'une écriture fragmentaire dans le roman Terrasse à Rome de Pascal Quignard?

7 En tant que procédé de création ou d'écriture, de quelle manière la fragmentation fonctionne-telle dans Terrasse à Rome ? Quelle est l'intentionnalité de la fragmentation dans Terrasse à Rome? Faut-il solliciter la collaboration du lecteur pour reconstituer l'histoire de Terrasse à Rome?

8 Raconter, c'est organiser un texte de longueur assez considérable; il faut faire en sorte que les évènements dépendent les uns des autres. Cependant, Pascal Quignard s'est montré décidé à tuer l'aventure dans le roman, à éliminer l'intrigue pour présenter seulement de simples séquences. Nous avons constaté que Terrasse à Rome bénéficie d'un subtil agencement. Loin de nouer et de dénouer une intrigue ; Pascal Quignard sait jouer entre les différents moments de l'histoire contée car il organise un système d'échos, d'annonce et de rappels qui assure la cohérence du texte (RAIMOND, 2000 : 106).

$9 \quad$ Les séquences narratives

10 Ainsi, le roman se caractérise par la succession et l'entrecroisement de séquences apparemment sans lien entre elles, en réalité solidement articulée car toutes les histoires tournent autour de Meaume, le personnage principal. Voici quelques exemples illustrations :

11 L'histoire de l'oreille: le lecteur découvre un fragment de cette histoire au début du roman, l'oreille en possession de Meaume : «Puis il sortit un flacon de son coffre. C'était une oreille humaine dans un bocal. Elle était devenue très pâle. Elle était aussi transparente que les mains que les grenouilles ont au bout de leurs bras» (38). Le lecteur saura seulement dans 
les pages 120-123-124-125, l'histoire entière de l'oreille et la raison pour laquelle elle figure dans l'inventaire de Meaume à Rome.

Le lecteur lit à la page 54 le premier fragment, du récit de l'évasion de l'ami de Meaume Abraham (le récit est présenté sans ordre chronologique): "Plus d'homme sur terre. Abraham et Meaume en profitent pour marcher à découvert ». Le lecteur retrouve la suite dans les pages 55-75-81-88-90 puis dans la page 140. Cette histoire est présentée par bribes et c'est au lecteur de relier les différents fragments.

Seulement l'histoire d'Abraham vacille entre le récit et le graveur c'est-à-dire que le lecteur ne sait plus s'il découvre le récit de l'évasion d'Abraham ou bien s'il s'agit seulement d'une description d'une gravure effectuée par Meaume.

Pascal Quignard brouille volontairement les pistes pour dérouter le lecteur.

Cependant de nombreuses versions entourent la mort de Meaume : à la page 107, chapitre 28 le lecteur saura que : " Meaume le graveur mourut à la fin de l'an1667 à Utrecht " (54-55). Et un peu plus loin le lecteur découvre qu'il s'est suicidé chez Honthorst. A la page 150, chapitre XLI, le lecteur apprendra aussi que Meaume agonisait avant sa mort : » Alors elle (Marie Aidelle) alla dans la chambre de Meaume le graveur, âgé de cinquante ans, agonisant et elle prit sa tête dans ses bras et le berça jusqu'à ce qu'il mourut. Ce fut ainsi qu'il rendit le souffle» (150). A la page 153 chapitre XLIII, nous lirons que Meaume est pris de délire avant de rendre l'âme :

Tandis que Meaume le graveur était encore de ce monde, dans les tous derniers jours qu'il vécut, mourant de faim, sa mémoire s'étant perdue, il ne reconnaissait plus les visages. Il faisait des gestes étranges sous son drap et il parlait aux mouches Meaume le graveur à la fin de sa vie connut plusieurs fatigues et souffrit d'embarras de pensée.

15 A la page 155 du même chapitre, Meaume a souffert de maladie avant sa mort, illustrons par cet extrait : « Le 24 , lors de la troisième vigile des natales, alors que tout le monde jeune, il meurt sans avoir rien pu déglutir depuis août ». Par ailleurs, le lecteur ne sait pas non plus pourquoi Marie Aidelle est venue rejoindre le graveur en Hollande, chez la belle -sœur de Gérard des Nuits, aux moments de son agonie. L'auteur nous fournit aussi deux versions qui justifient la raison de la présence de Marie Aidelle au chevet du mort, la première se trouve au chapitre XXVIII : « Le bel atelier d'Utrecht appartenait alors à l'épouse de Wihelm Van Honthorst dont le prénom était Catherine. C'est Marie Aidelle qui berce le cadavre de Meaume suicide chez Honthorst " (108). Le narrateur repose la question quarante pages plus loin, il nous révèle la raison de sa présence :

Le 16 décembre Meaume le graveur dicte un deuxième testament en -chuchotant davantage- à Utrecht. Sa gorge s'est définitivement serrée. Le notaire et Catherine Van Honthorst se tiennent penches sur le lit, leurs oreilles presque en contact avec sa bouche. Il ne mange plus depuis l'été. Le 18 il ajoute codicille où il met le nom de Marie Aidelle. C'est sans doute la raison pour laquelle Catherine Van Honthorst fait venir Marie. Il ne la reconnait pas (148).

16 Un autre récit déroute le lecteur : celui de la grossesse de Nanni. Le futur époux jaloux, Vanlacre, dont l'honneur a été bafoué, menace son rival et le contraint à quitter la ville. Or la jeune femme, Nanni, est enceinte. Personne, sauf elle sans doute, ne sait qu'il (l'enfant) l'est, non de l'époux, mais de l'amant, le graveur Meaume. Devenu vieux, celui-ci retrouvera son fils à son insu. Nous sommes en 1666. En 1640, il avait revu Nanni à Mayence et elle lui avait appris qu'elle était enceinte : 
Un an plus tard, en 1640 , il la vit (...). En réponse à l'une de ces questions, elle confirme qu'elle est mariée depuis dix mois. A une nouvelle question, elle répond que oui, elle a un petit. De qui ? Elle ne répond pas. Elle lève ses paupières. Elle rit. Elle prend sa main. » Viens », lui dit-elle. Il la regarde. Puis il fait non avec la tête. Il court. (32-33)

Et c'est vingt-huit chapitres plus tard que le lecteur a la réponse à la question de Meaume. C'est la confirmation de ce qu'il avait sans doute déjà compris avec l'attitude de Nanni (GODAR, 2006).

D'ailleurs, dès le début du roman, nous savons que le commis de Jakobsz, futur époux de Nanni, s'appelle Vanlacre :» Le commis de ruelle de Jakobsz qui s'appelle Vanlacre, s'est blessé en pulvérisant les carreaux de la fenêtre. » (21). Suite à une méprise, le fils qui tente de tuer Meaume prétend s'appeler Vanlacre:»Alors Meaume pleura tout à coup et se tourna vers le mur. Il demanda doucement, en flamand, dans la pénombre : » D'où viens-tu? »

-Brugge.

-Quel est ton nom?

-Vanlacre. » »(131).

19 Ce garçon est en quête de son vrai père qui refuse plus tard de le reconnaître. Meaume meurt de cette tentative d'assassinat. Et c'est son fils qui l'aura tué. L'intrigue est très simple. Mais elle ne se résume pas à un amour contrarié dont les conséquences sont irréversibles. Elle se fonde aussi sur l'existence d'un fils dont il n'est à priori pas question dans le roman. Vanlacre est un beau jeune homme : " il est extrêmement beau " (130), alors que son père défiguré est hideux. Pour Meaume, c'est compensation d'ordre narcissique car Vanlacre fait aussi partie de sa création.

Le roman commence et se clôt sur la vie de Meaume. Et cela indique peut-être un dispositif circulaire du roman. L'auteur nous présente différentes versions de la vie de Meaume en respectant le plus souvent les faits et les mêmes évènements vus de différents angles, la première version est faite par Meaume au début du roman :

Je suis né l'année 1617 à paris. J'ai été apprenti chez Follin à paris. Chez Rhuys le reforme dom à la cite de Toulouse chez Heemkers à Bruges. Après j'ai vécu seul. A Bruges j'aimais une femme et son visage fut entièrement brûlé. Pendant deux ans j'ai caché un visage hideux dans la falaise qui est au-dessus de Ravello en Italie ( 9).

Les deux autres versions sont reléguées à un narrateur extra diégétique. La première version se trouve à la page 159 :

Meaume le Graveur, Citoyen de la Ville de Rome apprit à dessiner chez Follin. Il apprit les rudiments du métier de cartier et les ombres auprès de Rhuys le Réformé à Toulouse. Il apprit la taille-douce et la technique de l'eau-forte chez Johann un Heemkers à Bruges (...) il eut le visage brûlé à l'eau-forte.

La deuxième version se trouve à la page 167. Il est important de tenir compte des variations entres les différentes versions puisque Pascal Quignard pousse le lecteur à se concentrer :

La grand-mère de Meaume baptisa l'enfant avec un doigt de sang de Concini quand on le déchire, pour le fortifier. Il aimait le vin noir, dont il abusait, il se laissa mourir. Meaume naquit à Paris au printemps de1617. Passe l'âge de cinquante ans, son visage était tendu et étrange. Il était très maigre (...) la blessure sur son visage ajoutait à l'incertitude de ses expressions. 
Même en peinture, le visage de Meaume est dissimulé. La perte du visage marque la fin d'un » je ", rendu à jamais illisible au regard de l'autre. Pour transcrire cette perte, le récit se fait littéral, lacunaire, fragmenté. Dans un court traité, Pascal Quignard rapporte ces mots de Flaubert à qui l'on proposait d'illustrer l'un de ses romans :» Vous voulez que le premier imbécile venu dessine ce que je me suis tué à ne pas montrer » (QUIGARD, 1990 : 131). En (d) écrivant les gravures, les mots vont bien montrer l'indicible, en passant par le burin et l'acide de Meaume. » La littérature est chaque mot. Son investigation propre est plus profonde à cause de l'image ", précisait Pascal Quignard dans "Rhétorique spéculative » (QUIGNARD, $2005:$ 12). La quête de cette image originaire lie bien sûr le fragment à la mémoire, dont les caractéristiques sont aussi celles du rêve et de l'image (HOUPPERMANS et al., 2005) :

Le hameau, la route, le pont, toutes les fermes et les étables sont sur cette gravure à la manière noire presque absorbées dans la ténèbre. Car l'ombre de la montagne projette une véritable ténèbre qui parait presque cramoisie à force d'être noire. Sauf un bout de route qui grimpe sur le flanc du pic d'en face. Un bout de route rose qui échappe à la couleur noire (55).

On retrouve des descriptions de gravures dans les pages 62-87-93-104-105-106-116-117-156-157-165-166.

Le lecteur est confronté à une chronologie pendant sa lecture, informé du avant et du après par le procédé d'analepse et de prolepse. Le roman se clôt sur l'incertitude, une incertitude de l'écriture, une incertitude de la lecture qui s'ajoute à l'incertitude des traits de Meaume : «La blessure sur son visage ajoutait à l'incertitude de ses expressions" (178). 
an contient, aussi, de scènes érotiques. Ces scènes érotiques servent (peut-être) de détente après l'effort de la lecture concentrée. Retenons l'exemple suivant :

Le rêve de Meaume est celui-ci : il est à dormir dans sa mansarde de Bruges (...) son sexe se dresse brusquement au-dessus de son ventre la lumière blanche épaisse torride du soleil ruisselle autour du buste d'une jeune femme blonde au long cou. La lumière déborde tous les contours de son corps, rongeant les silhouettes de ses joues et de ses seins. C'est Nannie Veet Jakobsz. Elle penche la tête. Elle s'assit sur lui. Elle le plonge en elle d'un coup il jouit. (44)

Pascal Quignard assimile la jouissance sexuelle au plaisir de lecture et explique les raisons de l'insertion des scènes sexuelles dans le roman :

La première personne n'est qu'un sexe masculin au repos et qui se recroqueville (...) Ce sexe peut émouvoir mais il ne peut pas transporter l'épouse, qui est le lecteur. Pour que le plaisir du texte demeure imprévisible, il faut que le lecteur ne puisse savoir d'où va venir le désir. Le désir ne peut dire je, ni avoir de visage ; il ne peut que désirer, bander. Fascinus fascinatus.

On retrouve d'autres scènes érotiques dans les pages 19-95-11-112-115.

Le roman contient aussi des lettres, de la correspondance. Retenons cet exemple : «La lettre de la fille de Jacob Veet Jakobsz adresse à Meaume : "j'ai reçu avec plaisir votre lettre qui demande des nouvelles qui soient de ma main. Elle témoigne de votre affection et vous en remercie " (25). Le chapitre V est une lettre de Nanni, réponse à celle qu'elle a reçue de Meaume et au portrait qu'il a fait d'elle. La lettre de Meaume ne nous est pas donnée à lire, mais on peut penser que c'était une lettre d'amour aux antipodes de la réponse qu'il reçoit ici, et par laquelle il apprend que le portrait qu'il a fait de la jeune fille a été amputé : elle a en effet (HOUPPERMANS et al., 2005) : « coupé aux ciseaux la gorge car vous l'aviez gravée nue et elle ne m'a pas paru décente » (26).

Les dialogues dans le roman sont particuliers par leurs formes courtes, (répliques sèches, aérées) renvoient souvent au niveau de langue familier. Citons quelques exemples : Le premier extrait c'est une conversation entre Meaume et Nanni :

Il faut que vous quittiez la ville aujourd'hui même.

-Pourquoi?

-Tout de suite.

-Pourquoi tout de suite? (29).

Le deuxième extrait c'est un dialogue entre Meaume et Marie Aidelle.

Ce sera difficile de vous coucher ici, dit Marie.

-Il y a toujours une écurie, dit Meaume.

-Non.

-Il y a toujours une grange.

-Non.

-Il y a toujours une étable.

-Oui.

-Cela suffira.

-Et pour travailler?

-Qu'est -ce qu'il faut ? Demanda la vielle Trognon qui était réapparue sous la grande poutre horizontale de la porte.

-Rien, rien (61).

Les dialogues créent de larges béances textuelles dans le roman. Le blanc désigne, dans la mise en page, chacun des intervalles, des espaces libres qui favorisent la lisibilité d'un texte. Les blancs dans Terrasse à Rome soulignent le rythme interne du texte. Ils créent un effet d'aération, de la suspension ou de dispersion à la fois visuel et 
sémantique (Larousse, 1992 : 42). Les répliques des personnages contribuent un créer un jeu de mots par le retour de la même expression » Il y a toujours une... ».

Remarquons aussi l'introduction de réflexion de plusieurs personnages : Marie, Aristoteles de Stagire, L'abbé de Saint- Cyran, Meaume, et le narrateur méditant sur le thème de la colère. Retenons à titre d'exemple les pensées de Marie et de Aristoteles de Stagire, illustrons par un extrait de la page 99 :

Sur la colère Marie disait: "Tous les malheureux sont nés d'une colère de leurs géniteurs que le plaisir qui a suivi n'a pas assouvie".

Dans la colère, nos oreilles cessent d'entendre.

Aristoteles de Stagire : "Il n'est pas plus possible à l'homme en colère d'arrêter sa fureur qu'au plongeur qui s'est élancé du rocher et stopper son élan et de ne pas atteindre l'eau".

A côté des pensées théoriques sur la production artistique, Quignard ajoute des pensées philosophiques.

\section{La difficulté de création}

Le roman comprend des réflexions sur l'art et la création artistique. Le narrateur tente par moment de fournir des explications et des clés de lecture pour faciliter le déchiffrage et la compréhension du texte. Citons l'exemple suivant :

Il (Meaume) appartenait à l'école des peintres qui peignaient dans une manière très raffinée les choses qui étaient considérées par la plupart des hommes comme les plus grossières: les yeux, les laboureurs, les coureurs de vase, les vendeurs de poudres de soudons, de crabes, de bras tachetés, de jeunes femmes qui se déchaussent, des jeunes femmes à peine habillées qui lisent qui lisent des lettres et qui rêvent d'amour, des servantes qui repassent des draps, tous les fruits murs ou qui commence de moisir et qui appellent l'automne, les déchets de repas, des beurriers, des tabagies, des joueurs de cartes (65).

La création artistique demande des efforts particuliers de concentration et de rigueur selon Meaume le graveur. Meaume nous soumet aussi ses difficultés de création. Citons à titre d'exemple cet extrait :

Dans le livre de Grüneghagen, dit que Meaume affirmait à la fin de sa vie : "Quand je m'assieds devant ma planche en cuivre le chagrin m'envahit. Je ne trouve plus le temps de songer à une image ou plutôt de la tenir devant mes yeux pour la reproduire, mon œuvre est ailleurs" (142-143).

La création d'une œuvre pure est la quête de Meaume comme tous les créateurs du monde. Grüneghagen, le biographe de Meaume rapporte aussi les propos de Meaume sur l'art. Illustrons par ce passage :

Grüneghagen rapporte ces propos de Meaume en 1652: "on dit regarder les graveurs comme des traducteurs qui font passer les autres d'une long riche et magnifique dans une autre que les mains à la vérité mais qui a plus la violence. Cette violence impose aussitôt son silence à celui qui est confronté" (163).

L'auteur n'hésite pas non plus de nous dévoiler la méthode de travail de Meaume et la spécificité de la gravure de cet artiste. Voici un extrait : «La manière noire est une gravure à l'envers » (93). Meaume s'ingénie à produire une gravure à l'envers au même titre que Pascal Quignard écrit un roman à l'envers. Pascal Quignard met en valeur le génie créateur de Meaume qui peut produire un chef-d'œuvre en s'inspirant et exploitant des éléments banals et insignifiants à l'instar de l'écrivain de ce roman qui écrit un subtil roman à partir d'une histoire simple, banale voire ordinaire (la vie de Meaume). 
N'existe-t-il pas une ressemblance entre l'activité créatrice du graveur et celle de l'écrivain?

L'art de Quignard est d'embrasser plusieurs arts en même temps: il évoque une gravure, il narre une action en train de se produire, puis évoque une allégorie de la création artistique.

Le roman de Pascal Quignard, est polyptique. Terrasse à Rome présente un aspect singulier, une forme de discontinue que Dominique Viart qualifie de "syncope ", une forme elliptique, fragmentaire, entrecoupée de silence (TURIN, 2009 : 138) et de blancs.

Pascal Quignard présente un "roman-jeu » ou un « roman-puzzle », au sujet " prétexte et mince ». C'est aussi une mise en crise du langage car l'écriture de ce roman se conteste donc elle-même et prévient, en somme, les lecteurs qui pourraient la trouver illisible ou déroutante. Dans ce sens Dominique Viart souligne : « fiction et réflexion travaillent ensemble » (VIART, 2004) dans le roman de Pascal Quignard.

Il est probable que chez Pascal Quignard, la pratique de cette écriture éclatée, problématique, ne s'explique pas simplement par un effet de mode ou par gout de la virtuosité, mais coïncide étroitement avec la crise intérieure que traverse l'auteur. Le brouillage du récit semble correspondre aux contradictions du réel et à la difficulté de les résoudre. » Le roman n'est plus le miroir qu'on promène le long d'une route, mais le miroir brisé d'un trouble intérieur » (TONNET LACROIX, 2003 : 171-172).

De fait, les écrivains actuels tentent souvent d'esquiver la forme romanesque, lui cherchent des alternatives, la reprennent de façon parodique ou ludique, stratégies qui témoignent bien de la permanence du soupçon à son égard, mais également d'une forme de nostalgie. Car la grande question qui se pose à eux, c'est de savoir comment dépasser « l'ère du soupçon » sans pour autant revenir à la " naïveté » antérieure. S'ils n'ont plus à déjouer les ruses naïves du romancier traditionnel, ils veulent prendre leurs distances avec les ruses plus sophistiquées du Nouveau Romancier. Autrement dit, ils désirent échapper aux catégories du traditionnel et du moderne. Ces attitudes d'esquive, ou de jeu, sont sans doute le signe de «l'épuisement » du roman, mais peut être aussi celui de son « renouvellement $»^{1}$.

6 Il est encore une façon d'esquiver le roman et ses conventions, c'est d'adopter des formes souples, parfois fragmentaires, où le fil narratif peut être constamment rompu par les digressions et où se mêlent récit, journal, essai, réflexion, fantasmes, rêves, contes. C'est en effet le difficile rapport à l'écriture, qui est au centre de ce roman. Parfois, on dirait que le récit ne "prend" pas. «Il apparait par bribes, il s'encombre d'écarts, de variations, où prime plus finalement la voix narrative que l'"action" elle-même» (TONNET LACROIX, $2003: 275$ ).

D'ailleurs, la remarque de Gracq sur » l'existence simultanée de deux littératures de qualité d'un côté une littérature de rupture (...) - et de l'autre une littérature de tradition ou de continuité » (Ibid : 175) semble donc se vérifier dans le roman de Pascal Quignard. Pascal Quignard n'en revient pas aux formes littéraires traditionnelles. Plus juste est de considérer qu'effectivement sujet et récit font retour sur la scène culturelle, mais sous la forme de questions insistantes, de problèmes irrésolus, de nécessités impérieuses (VIART et VERCIER, 2005).

Pour Quignard, il s'agit de se servir des formes et des structures de la fiction pour mettre en exergue le travail, ou le jeu de la narration. Il veut intéresser le lecteur moins à l'histoire relatée qu'à l'habilité de l'écrivain et à difficulté de l'acte créatif. Terrasse à 
Rome se présente comme une réflexion sur les structures narratives. La subversion narrative détruit l'édifice romanesque mais laisse la possibilité d'une reconstruction offrant une ouverture à la réflexion du lecteur.

Le roman est incertain, l'écriture en défaut, alors: "A époque incertaine, romans incertains, romans indécidables" (BLANCKEMAN, 2000). Dans le corpus d'étude, ce récit développe une trame narrative, mais celle-ci se trouve décalée par rapport à un référent, dissimulée dans un texte. Pour Pascal Quignard, il s'agit de se servir des formes et des structures de la fiction pour valoriser l'écriture: Pascal Quignard veut intéresser le lecteur moins à l'histoire racontée qu'à la virtuosité de l'écrivain.

Peut-on parler d'une écriture subversive dans le roman Terrasse à Rome de Pascal Quignard?

Le récit moderne, effectivement, vise à la disparition de l'intrigue au profit du récit, de l'écriture.

Pascal Quignard considère l'élaboration de son œuvre comme un lent et patient cheminement, une difficile mais constante progression. N'accordant aucun crédit à des notions telles que l'inspiration, le talent ou le génie, il ne croit qu'en une seule vertu » le travail » : «Par la manière noire chaque forme sur la page semble sortir de l'ombre comme un enfant du sexe de sa mère» (94). L'œuvre est et n'est que le fruit du labeur de son créateur. Son travail d'écriture est sans doute réellement obstiné, méticuleux, et répétitif (GENIN, 1997).

53 En conclusion, on peut dire que le souci de Pascal Quignard est une interrogation constante sur: comment pourrait-il raconter une histoire vraiment nouvelle? comment pourrait-il la raconter selon les modalités qui n'auraient pas encore été employées? L'histoire dans Terrasse à Rome est un prétexte à l'écriture, à la recherche de ce qui est perdu. » On travaille avec le langage pour se comprendre et s'éloigner du langage ", analyse Pascal Quignard.

54 En effet, nul doute qu'il aurait été aisé pour le lecteur de Pascal Quignard de reconnaitre à travers cette évocation un équivalent de la pratique cut-up si souvent employé par cet auteur.

Cette technique consiste en la rupture de l'unité de ton qui s'est instaurée au sein

d'un épisode par l'insertion mal à propos d'un détail saugrenu ou dans le brouillage

de la linéarité dont procède la fiction par l'intrusion hors de propos d'un segment textuel inattendu (Idem).

On peut retenir le fait de savoir cerner dans sa création romanesque une très subtile poétique du fragment.

Ce qui retient désormais l'attention, ce n'est certes plus l'histoire, mais les mots pour le dire, «les à-côtés du récit ", si bien que le texte narratif devient un texte poétique et substitue à l'intérêt romanesque, l'intérêt esthétique (OUHIBI GHASSOUL, 2004).

L'anti- conformisme de ce roman, constaté dans l'atomisation, la singularité des écritures, originalité que nous avons montrée, en nous écartant de toute tentation aprioriste, idéologique ou autre, en gardant à l'esprit, la sauvegarde de la spécificité de ce texte littéraire.

Ce romancier, tout en innovant et tout en refusant les sentiers battus, conserve à l'écriture son statut habituel, c'est-à-dire normatif et s'en prennent à l'organisation du récit.

Terrasse à Rome met en relief 
deux référents qui se superposent, l'un fictionnel, l'autre textuel. Systématisant la pratique gidienne, le récit donne à lire, dans le temps même de la narration, sa narrativité. Les écrivains inventent le passé : l'idée même de littérature appelle cette fabrication et cette fabulation rétrospectives. Quand les repères canoniques se mettent en cause, l'écriture se met en jeu : elle mérite et médite un héritage qui ne lui est plus nécessairement acquis. (BLANCKEMAN, 2000, 211). d'affranchissement narratologique, que multiplient les récits portés par une écriture à grande vitesse, à très forte rythmique, marquent une volonté similaire : fuir les états constitués, faire bouger les contours admis et les profils fixes, répercuter organiquement les mutations du contemporain, en élaborant ainsi un sens adapté. Cette déconstruction se manifeste par un éclatement des catégories du récit, une dispersion diégétique, une dissolution des référents stables. Bruno Blanckeman souligne aussi que

le récit réinstalle l'homme dans des histoires, en proposant des fictions renouvelées, ouvertes au désordre du réel, à la combinatoire des mots, à la structuration du sens. Points de société, parcelles d'humanité, options d'existence appellent sans discrimination, d'une œuvre à l'autre, la forme littéraire appropriée fut-elle hybride (BLANKEMAN, 2000, 211).

Le romancier retenu pour cette étude à savoir Pascal Quignard a séduit, pour son professionnalisme du récit, sa technicité du roman, son expérimentation de l'écriture, son écriture a- canonique. Pour Alain Robbe -Grillet :

Ce qui fait la force du romancier, c'est justement qu'il invente en toute liberté sans modèle. Le récit moderne a ceci de remarquable : il affirme de propos délibéré ce caractère, à tel point même que l'invention, l'imagination, deviennent à la limite le sujet du livre. (ROBBE-GRILLET, $1968: 30$ )

Pascal Quignard a la capacité d'ouvrir plusieurs pistes narratives et à les brouiller en laissant le lecteur choisir, comparer et réfléchir. Il faut solliciter, comme Gide le voulait déjà, la collaboration du lecteur, il suffit de lui proposer les éléments d'un puzzle avec lesquels il puisse s'amuser à exercer la liberté de son intelligence et de son imaginaire pour reconstituer le récit. Terrasse à Rome est, en somme, comme une longue méditation portant sur le travail de l'écrivain et la création artistique.

\section{BIBLIOGRAPHIE}

ASSELIN, I., Le roman fragmenté, Presses universitaires de Rennes, 2003.

BERTRAND, M., Langue romanesque et parole scripturale, Littérature PUF, 1987. 
BLANCKEMAN, B., Les récits indécidables. Presse universitaire de Sentension. Paris 2000.

GENIN, C., Lecture studieuse et lecture poignante, Edition honore, Champion Paris, Paris, 1997.

GODAR, R., Itinéraires du roman contemporain, Armand Colin, 2006.

HOUPPERMANS, S., BOSMAN DELZONS, C., DE RUYNTER-TOGNOTTI, D., Territoires et terres

d'Histoires perspectives, horizons, jardins secrets dans la littérature française d'aujourd'hui, Edité par Sjef Houppermans, Christine Bosman Delzons, Danièle de Ruynter- Tognotti. Amsterdam, New York, Ny 2005, Editions Rodopi B. N, Amsterdam-New York 2005.Printed in the Netherlands.

RAIMOND, M., Le roman, Armand Colin, Paris 1987-2000.

ROUSSEL-MEYER, M., La fragmentation dans le roman, Presses universitaires Blaise Pascal, Clermont -Ferrand, 2011.

OUHIBI GHASSOUL, B., Perspectives critiques : le roman algérien de langue française dans la décennie 1985-1995. Thèse de doctorat d'état 2003-2004. Oran. P. 117-118-119-129.

ROBBE-GRILLET, A., Pour un nouveau roman, Gallimard, 1968.

TURIN, G., Entre centre et absence : fragmentation et style chez Pascal Quignard, Dans littérature, Edition Armand Colin, 2009.

VIART, D., » Les fictions critiques » de Pascal Quignard, Etudes françaises, Volumes40, Number2, 2004.

QUIGNARD, P., » Terrasse à Rome », Gallimard, 2000.

NOTES

1. Selon les formules J. Barth à propos du roman américain depuis les années 80 , «The literature of exhaustion" 1967, et "The literature of replenishment"1980, dans la revue américaine "The Atlantic", ce dernier texte repris dans "Poétique", n 48 , nov. 1981. Cité dans le livre Tonnet Lacroix (275).

\section{RÉSUMÉS}

Pascal Quignard publie Terrasse à Rome en 2000. Le roman contient des descriptions de tableaux, de gravures, d'incessants sauts chronologiques, des réflexions. Mais aussi des énigmes, des bribes de récits, des thèmes qui se faufilent entre les chapitres. La composition de ce roman a exigé de l'auteur une parfaite disposition de chacune des scènes, de chacun des mots, en fonction de la totalité textuelle. L'intégration mal à propos d'un seul élément entraîne la rupture du dispositif général et /ou l'écroulement de la structure romanesque. Il faut solliciter, comme Gide le voulait déjà, la collaboration du lecteur, il suffit de lui proposer les éléments d'un puzzle avec lesquels il puisse s'amuser à exercer la liberté de son intelligence et de son imaginaire. Terrasse à Rome est comme une longue méditation portant sur le travail de l'écrivain et la création artistique. 
Pascal Quignard published Terrasse à Rome in 2000. The novel contains descriptions of paintings, engravings, incessant chronological jumps, and reflections. It also includes enigmas, snippets of stories, themes that weave between chapters. The composition of this novel required from the author a perfect disposition of each of the scenes, of each of the words, according to the textual totality. The improper integration of a single element leads to the breakdown of the general device and / or the collapse of the novelistic structure. It is necessary to solicit, as Gide already wanted it, the collaboration of the reader; it is enough to propose to him the elements of a puzzle with which he can have fun exercising of the freedom of his intelligence and his imagination. Terrace in Rome is like a long meditation on the writer's work and artistic creation.

INDEX

تجزئة, كتابة, تدمير, بناء, قارئفهرس الكلمات المفتاحية:

Keywords : fragmentation, writing, destruction, construction, reader

Mots-clés : fragmentation, écriture, destruction, construction, lecteur

\section{AUTEUR}

\section{NABILA BEKHEDIDJA}

Université Oran 2 